Proc. Estonian Acad. Sci. Biol. Ecol., 2005, 54, 1, 53-66

\title{
Annotated list of rotifers of Lake Võrtsjärv
}

\author{
Taavi Virro $^{\mathrm{a} *}$ and Juta Haberman ${ }^{\mathrm{b}}$ \\ ${ }^{\text {a }}$ Institute of Zoology and Hydrobiology, University of Tartu, Vanemuise 46, 51014 Tartu, Estonia \\ ${ }^{b}$ Võrtsjärv Limnological Station, Institute of Zoology and Botany, Estonian Agricultural University, \\ 61101 Rannu, Tartumaa, Estonia; juta@zbi.ee
}

Received 3 December 2004, in revised form 17 January 2005

\begin{abstract}
The present review makes an inventory of published information on the taxonomic composition of rotifers in L. Võrtsjärv, Estonia, since 1920, and provides a revised list of rotifers recorded from the lake, with up-to-date taxonomy. The list of synonyms used in earlier literature is also given. A total of 173 rotifer taxa (138 species) belonging to 22 families and 46 genera have so far been recorded from L. Võrtsjärv. The most taxon-rich family is Brachionidae with 41 taxa listed, followed by Synchaetidae with 19 and Trichocercidae with 18 taxa. The most diverse genera are Trichocerca (17 taxa) and Lecane (14). Most (75\%) of the rotifer taxa found have cosmopolitan or wide distribution, approximately $11 \%$ have Holarctic and $2 \%$ Palaearctic occurrence. The rotifer fauna of L. Võrtsjärv includes four rare species: Cephalodella compacta, Eosphora thoides, Monommata grandis, and Resticula gelida.
\end{abstract}

Key words: Rotifera, taxonomic composition, shallow eutrophic lake.

\section{INTRODUCTION}

Rotifers constitute an important part of the zooplankton in strongly eutrophic L. Võrtsjärv. Their abundance fluctuates between 163000 and $2130000 \mathrm{ind} \mathrm{m}^{-3}$ during the year, the average is $656000 \mathrm{ind}^{-3}$. The share of rotifers in the zooplankton abundance is great all the year round (82\%), being especially high in winter (99\%) and spring (90\%). In summer and autumn, owing to abundant occurrence of cladocerans and copepods, the share of rotifers diminishes, remaining still on a rather high level $(68 \%)$. The biomass of rotifers is low ranging $0.112-0.858 \mathrm{~g} \mathrm{~m}^{-3}$, with an average $0.278 \mathrm{~g} \mathrm{~m}^{-3}$. They form quite a considerable part $(28 \%)$ of the zooplankton biomass during the vegetation period. In the winter zooplankton, rotifers are the main component $(91 \%)$ in the zooplankton biomass (Haberman \& Virro, 2003).

*Corresponding author, tvirro@ut.ee 
The first data on the rotifers of L. Võrtsjärv were published in the monograph by Mühlen \& Schneider (1920). In this study, based on the samples collected during 1911-1913, 26 rotifer taxa were listed. In 1952-1954, after an interval of almost 40 years, the zooplankton of the lake was investigated again by Schönberg (1958). Only the dominating four rotifer species were mentioned among 63 zooplankters. Since 1964, the zooplankton of L. Võrtsjärv has been monitored monthly. Together with this, more data began to accumulate on rotifers. Haberman \& Mäemets (1973) summarized earlier literature data and the investigations made during 1954-1966, providing a list of zooplankton taxa with 51 rotifer taxa (45 species). The pelagic rotifer community was studied in detail by Haberman $(1976,1978)$. She reported 34 taxa (31 species) of rotifers from the material collected in 1965-1966. In the 1970s, the studies were carried on dealing mostly with the seasonal dynamics and quantitative aspects of rotifers as a group and of dominant species. In 1980-1982, the pelagial and littoral habitats of L. Võrtsjärv were investigated by Kutikova \& Haberman (1986), recording 148 rotifer taxa (129 species). The same taxa are mentioned in further rotifer or general zooplankton works based on the analysis of monthly monitoring data from the years 1989-1997 (e.g. Haberman, 1995, 1998; Haberman \& Põllumäe, 1998). More recently, the previous information has been summarized and supplemented with new data from different sets of samples dating up to 2002. Further 18 taxa (6 species) have been added to the list of rotifers previously known from L. Vorrtsjärv (Haberman \& Virro, 2003; Virro \& Haberman, submitted).

The aim of the present review is to make an inventory of all available information published on the taxonomic composition of rotifers in L. Võrtsjärv since 1920 and to provide a revised list of rotifers recorded from the lake, with up-to-date taxonomy. Summarized and improved information on the taxonomic composition is essential for the monitoring of the compositional changes in the rotifer fauna of L. Võrtsjärv, for the assessment of its ecological status, and conservation of biodiversity, as well as for facilitating future checkings of the earlier lists.

\section{DESCRIPTION OF THE LAKE}

Lake Võrtsjärv is situated in central Estonia. It is the second largest lake in the Baltic region. Data on several morphometric and physico-chemical characteristics are presented in Table 1. Based on the long-term nutrient data from the years 1968-2001, L. Võrtsjärv can be qualified as strongly eutrophic, its southern part even hypertrophic. The shallowness of the lake and the waveinduced resuspension of bottom sediments contribute to the formation of high seston (detritus) concentration and high turbidity during summer. Algal blooms are common in the lake. Filamentous algae are dominating all the year round: species of Aulacoseira in spring, Limnothrix planktonica (Wołosz.) Meffert, L. redekei (van Goor) Meffert, Planktolyngbya limnetica (Lemm.) Kom.-Legn., and Aphanizomenon skujae Kom.-Leng. et Cronb. in summer and autumn 
Table 1. Morphometric and physico-chemical characteristics (mean values) of L. Võrtsjärv

\begin{tabular}{lc}
\hline \multicolumn{1}{c|}{ Characteristic } & Value \\
\hline Length, km & 35 \\
Maximum width, km & 15 \\
Mean surface area, $\mathrm{km}^{2}$ & 270 \\
Mean depth, m & 2.8 \\
Maximum depth, m & 6.0 \\
Mean volume, $\mathrm{km}^{3}$ & 0.75 \\
Mean annual range of water level fluctuations, m & 1.4 \\
Mean residence time, year & 1 \\
Transparency, m & 1.07 \\
pH & 8.1 \\
Total P, mg P m ${ }^{-3}$ & 52 \\
Total N, mg N m ${ }^{-3}$ & 1540 \\
Chl $a$, mg m & \\
Duration of ice cover, day & 22 \\
Maximum water temperature, ${ }^{\circ} \mathrm{C}$ & 135 (November-April) \\
\end{tabular}

(Nõges et al., 2003). Zooplankton is dominated by small cladocerans (Chydorus sphaericus (Müller), Bosmina longirostris (Müller)) and rotifers typical of eutrophic waterbodies (Haberman \& Virro, 2003). The total number of bacteria fluctuated from $0.28 \times 10^{6}$ to $11 \times 10^{6}$ cells $\mathrm{mL}^{-1}$ during the period of $1963-2001$, being as an average $3.7 \times 10^{6}$ cells $\mathrm{mL}^{-1}$ (Tammert \& Kisand, 2003).

\section{MATERIAL AND METHODS}

The present review is based on all previous taxonomical or ecological publications dealing with rotifers of L. Vorrtsjärv to a greater or lesser extent. The general classification scheme followed in the present list is that used by Melone et al. (1998). The arrangement of families is based on Kutikova (1970) with modifications after the updated keys published in the series Guides to the Identification of the Microinvertebrates of the Continental Waters of the World (see the citations in the text). The genera and species within each family are arranged in alphabetical order. Infrasubspecific forms (ecotypes or morphotypes) are included. Although they have no systematic status, they can be significant from the ecological aspect, the more so because in many cases the status of these infrasubspecific units is not yet clear. The nomenclature of Lecanidae, Notommatidae, Scaridiidae, Proalidae, Dicranophoridae, Asplanchnidae, Gastropodidae, Lindiidae, Synchaetidae, and Trochosphaeridae follows the Guides ... (Segers, 1995; Nogrady et al., 1995; De Smet, 1996; De Smet \& Pourriot, 1997; De Paggi et al., 2002). In the other cases various sources have been consulted, including Donner (1965), Kutikova (1970), Koste (1978), Koste \& Shiel (1987, 1989a, 1989b), and Shiel \& Koste (1992). 


\section{RESULTS AND DISCUSSION}

A total of 173 rotifer taxa (138 species) belonging to 22 families and 46 genera have so far been recorded from L. Võrtsjärv (Table 2). The list of synonyms used in earlier literature is given in Table 3. The most taxon-rich family is Brachionidae with 41 taxa (21 species) listed, followed by Synchaetidae with 19 (18) and Trichocercidae with 18 (16) taxa. The most diverse genera are Trichocerca (17 taxa, 15 species) and Lecane (14 taxa, 14 species). Most (75\%) of the rotifer taxa found have cosmopolitan or wide distribution, approximately $11 \%$ have Holarctic and 2\% Palaearctic occurrence. Five taxa (3\%) are presently known only from Europe: Collotheca balatonica, Dicranophorus robustus f. europaeus, Encentrum eurycephalum, Polyarthra dissimulans, and Trichotria pocillum bergi.

Table 2. Systematic list of Rotifera recorded from Lake Võrtsjärv, with remarks on occurrence. Key to reference sources: $1=$ Mühlen \& Schneider, 1920; $2=$ Haberman \& Mäemets, 1973; $3=$ Haberman, 1978; $4=$ Kutikova \& Haberman, 1986; $5=$ Haberman, 1998; $6=$ Haberman \& Virro, 2003; 7 = Virro \& Haberman, submitted. Pe = pelagial plankton; Li = littoral plankton; Ps $=$ psammon; $?=$ probable presence

\begin{tabular}{l}
\hline \multicolumn{1}{c}{ Taxon } \\
\hline \multicolumn{1}{c}{ Phylum Rotifera } \\
Superclass Eurotatoria \\
Class Bdelloidea
\end{tabular}

\section{Class Monogononta}

\section{Order Ploimida}

Family Notommatidae

Cephalodella compacta Wiszniewski, 1934

C. gibba (Ehrenberg, 1832)

C. gibba (Ehrenberg, 1832) f. microdactyla

Koch-Althaus, 1963

C. megalocephala (Glasscott, 1893)

C. sterea (Gosse, 1887) f. minor Donner, 1950

C. ventripes (Dixon-Nuttall, 1901)

Eosphora thoides Wulfert, 1935

Monommata grandis Tessin, 1890

Notommata cyrtopus Gosse, 1886

N. diasema Myers, 1936

Resticula gelida (Harring et Myers, 1922)

$\begin{array}{lll}4,5,6 & 7 & \mathrm{Li} \\ 4,5,6 & 6 & \mathrm{Ps} \\ 4,5,6 & 6 & \mathrm{Pe}, \mathrm{Li} \\ 4,5,6 & 6 & \mathrm{Li} \\ 4,5,6 & 6 & \mathrm{Ps} \\ 4,5,6,7 & 1,4-6,8-12 & \mathrm{Pe}, \mathrm{Li}\end{array}$

$\begin{array}{lll}4,5,6 & 8 & \mathrm{Ps} \\ 4,5,6 & 6-11 & \mathrm{Li}, \mathrm{Ps} \\ 6 & 4 & \mathrm{Pe} \\ 4,5,6 & 6 & \mathrm{Ps} \\ 4,5,6 & 6 & \mathrm{Ps} \\ 4,5,6 & 7 & \mathrm{Li} \\ 4,5,6 & 4 & \mathrm{Li} \\ 4,5,6 & 9 & \mathrm{Li} \\ 4,5,6 & 6 & \mathrm{Ps} \\ 4,5,6 & 8 & \mathrm{Ps} \\ 4,5,6,7 & 3 & \mathrm{Li}\end{array}$


Table 2. Continued

\begin{tabular}{c|c|c|c}
\hline Taxon & Source & Month & Habitat \\
\hline
\end{tabular}

Family Scaridiidae

Scaridium longicaudum (Müller, 1786)

\section{Family Trichocercidae}

Elosa spinifera Wiszniewski, 1932

Trichocerca brachyura (Gosse, 1851)

T. capucina (Wierzejski et Zacharias, 1893)

T. dixon-nuttalli (Jennings, 1903)

T. elongata (Gosse, 1886)

T. jenningsi Voigt, 1956

T. longiseta (Schrank, 1802)

T. porcellus (Gosse, 1886)

T. porcellus (Gosse, 1886) f. major (Hauer, 1935)

T. pusilla (Lauterborn, 1898)

T. rattus (Müller, 1776)

T. rattus (Müller, 1776) f. carinata (Ehrenberg, 1830)

T. rousseleti (Voigt, 1902)

T. ruttneri Donner, 1953

T. similis (Wierzejski, 1893)

T. stylata (Gosse, 1851)

T. taurocephala (Hauer, 1931)

T. tenuior (Gosse, 1886)

\section{Family Gastropodidae}

Ascomorpha ecaudis Perty, 1850

A. saltans Bartsch, 1870

Gastropus stylifer Imhof, 1891

\section{Family Synchaetidae}

Ploesoma hudsoni (Imhof, 1891)

Polyarthra dissimulans Nipkow, 1952

P. dolichoptera Idelson, 1925

P. cf. dolichoptera Idelson, 1925

P. euryptera Wierzejski, 1891

P. longiremis Carlin, 1943

P. luminosa Kutikova, 1962

P. major Burckhardt, 1900

P. minor Voigt, 1904

$P$. remata Skorikov, 1896

P. vulgaris Carlin, 1943

Synchaeta grandis Zacharias, 1893

S. kitina Rousselet, 1902

S. lakowitziana Lucks, 1930

S. longipes Gosse, 1887

S. oblonga Ehrenberg, 1831

S. pectinata Ehrenberg, 1832

S. stylata Wierzejski, 1893

S. verrucosa Nipkow, 1961 $\begin{array}{lll}4,5,6 & 7 & \mathrm{Li}\end{array}$

$\begin{array}{lll}4,5,6 & 8 & \mathrm{Li} \\ 6,7 & 1,7 & \mathrm{Pe} \\ 1,2,3,4,5,6 & 5-10 & \mathrm{Pe}, \mathrm{Li} \\ 6 & 7 & \mathrm{Pe} \\ 4,5,6,7 & 12 & \mathrm{Li} \\ 4,5,6 & 6 & \mathrm{Li} \\ 2,4,5,6 & 7 & \mathrm{Li} \\ 4,5,6 & 6,9 & \mathrm{Li} \\ 6 & 7 & \mathrm{Pe} \\ 4,5,6 & 6-9 & \mathrm{Pe}, \mathrm{Li} \\ 4,5,6 & 9 & \mathrm{Li} \\ 4,5,6 & 6-8 & \mathrm{Li} \\ 4,5,6 & 6-9 & \mathrm{Pe}, \mathrm{Li} \\ 4,5,6 & 6,7 & \mathrm{Li} \\ 4,5,6 & 8 & \mathrm{Pe} \\ 4,5,6 & 7 & \mathrm{Pe} \\ 4,5,6 & 8 & \mathrm{Ps} \\ 4,5,6 & 6,7 & \mathrm{Pe}, \mathrm{Ps}\end{array}$

$\begin{array}{lll}4,5,6 & 6 & \mathrm{Li} \\ 6 & 6 & \mathrm{Pe} \\ 2,3,4,5,6 & 5-10 & \mathrm{Pe}, \mathrm{Li}\end{array}$

$1,2,3,4,5,6 \quad 4-10 \quad \mathrm{Pe}, \mathrm{Li}$

$2,3,6$

1?, 2, 3, 4, 5, 6, 7 1-7, 10-12 $\mathrm{Pe}, \mathrm{Li}$

$6 \quad 4-7 \quad \mathrm{Pe}, \mathrm{Li}$

$1,4,5,6 \quad 6-8 \quad \mathrm{Pe}, \mathrm{Li}$

$\begin{array}{lll}4,5,6 & 6,8 & \mathrm{Li}\end{array}$

$2,3,4,5,6 \quad 5-10 \quad \mathrm{Pe}, \mathrm{Li}$

$1 ?, 4,5,6 \quad 7-10 \quad \mathrm{Pe}, \mathrm{Li}$

1?, 2, 3, 4, 5, 6 6, $7 \quad \mathrm{Pe}, \mathrm{Li}$

1?, 2, 3, 4, 5, 6, 7 3, 5-7, $9 \quad \mathrm{Pe}, \mathrm{Li}$

$1 ?, 2,3,4,5,65,7 \quad \mathrm{Pe}, \mathrm{Li}$

$4,5,6 \quad 5-10 \quad \mathrm{Pe}, \mathrm{Li}$

$4,5,6 \quad 4-8,10 \quad \mathrm{Pe}, \mathrm{Li}$

$7 \quad 1 \quad \mathrm{Pe}$

$4,5,6 \quad 4,6 \quad \mathrm{Li}$

$4,5,6 \quad 4-9 \quad \mathrm{Pe}, \mathrm{Li}$

4, 5, $6 \quad 4-7,9-11 \quad \mathrm{Pe}, \mathrm{Li}$

$4,5,6 \quad 5-9 \quad \mathrm{Pe}, \mathrm{Li}$

4, 5, 6, $7 \quad 1-5,10-12 \quad \mathrm{Pe}, \mathrm{Li}$

Continued overleaf 
Table 2. Continued

\begin{tabular}{c|c|c|c}
\hline Taxon & Source & Month & Habitat \\
\hline
\end{tabular}

\section{Family Lindiidae}

Lindia truncata (Jennings, 1894)

$4,5,6 \quad 8$

$\mathrm{Li}$

\section{Family Dicranophoridae}

Albertia naidis Bousfield, 1886

Aspelta sp.

Dicranophorus hercules Wiszniewski, 1932

$1,2,6$

$4,5,6$

$4,5,6$

D. hercules Wiszniewski, 1932 f. adenta Wulfert, 1961

D. luetkeni (Bergendal, 1892)

$4,5,6$

D. robustus Harring et Myers, 1928 f. europaeus

$4,5,6$

$4,5,6$

8

Wulfert, 1936

Dicranophorus sp.

Encentrum eurycephalum Wulfert, 1936

E. marinum (Dujardin, 1841)

Wierzejskiella sabulosa (Wiszniewski, 1932)

W. velox (Wiszniewski, 1932)

$\begin{array}{lll}6 & 5 & \text { Pe } \\ 4,5,6 & 6 & \text { Ps } \\ 4,5,6 & 8 & \text { Ps } \\ 4,5,6 & 6 & \text { Ps } \\ 4,5,6 & 6 & \text { Ps }\end{array}$

\section{Family Asplanchnidae}

Asplanchna girodi de Guerne, 1888

A. herrickii de Guerne, 1888

$\begin{array}{lll}4,5,6,7 & 1-4,9,12 & \mathrm{Pe}, \mathrm{Li} \\ 1,2,3,4,5,6 & 6-9 & \mathrm{Pe}, \mathrm{Li} \\ 1,2,3,4,5,6,7 & 1-12 & \mathrm{Pe}, \mathrm{Li} \\ 4,5,6 & 5-8,10,11 & \mathrm{Pe}, \mathrm{Li}\end{array}$

A. priodonta Gosse, 1850 f. helvetica Imhof, 1884

$\begin{array}{lll}4,5,6 & 4-8 & \mathrm{Pe}, \mathrm{Li} \\ 4,5,6 & 10 & \mathrm{Pe} \\ 2,3,4,5,6 & 6,8 & \mathrm{Pe}, \mathrm{Li}, \mathrm{Ps} \\ 1,2,6 & 7 & \\ 4,5,6 & 6 & \mathrm{Pe} \\ 4,5,6 & 4-6 & \mathrm{Li} \\ 4,5,6 & 8 & \mathrm{Li} \\ 4,5,6 & 6 & \mathrm{Ps} \\ 4,5,6 & 7,8 & \mathrm{Li} \\ 1,2,3,4,5,6,7 & 1,5-9,12 & \mathrm{Pe}, \mathrm{Li} \\ 4,5,6 & 4-7,10 & \mathrm{Li} \\ 4,5,6 & 8 & \mathrm{Li} \\ 4,5,6 & 8 & \mathrm{Ps} \\ 4,5,6 & 7,8 & \mathrm{Pe}, \mathrm{Li}\end{array}$

\section{Family Proalidae}

Bryceella stylata (Milne, 1886)

$\begin{array}{lll}4,5,6 & 8 & \text { Ps }\end{array}$

\section{Family Epiphanidae}

Proalides tentaculatus Beauchamp, 1907 f. wulferti Sudzuki, 1959

$4,5,6 \quad 8 \quad \mathrm{Pe}$


Table 2. Continued

\begin{tabular}{c|c|c|c}
\hline Taxon & Source & Month & Habitat \\
\hline
\end{tabular}

\section{Family Trichotriidae}

Trichotria pocillum (Müller, 1776)

T. pocillum bergi (Meissner, 1906)

$\begin{array}{lll}1,2,4,5,6 & 6-11 & \mathrm{Pe}, \mathrm{Li} \\ 4,5,6 & 5,6,9,10 & \mathrm{Pe}, \mathrm{Li} \\ 1,2,3,4,5,6 & 4,5,7,9 & \mathrm{Pe}, \mathrm{Li} \\ 4,5,6 & 8 & \mathrm{Li} \\ 4,5,6 & 9,10 & \mathrm{Pe}, \mathrm{Li} \\ 4,5,6 & 8-10 & \mathrm{Pe}, \mathrm{Li}\end{array}$

T. tetractis (Ehrenberg, 1830)

T. tetractis (Ehrenberg, 1830) f. caudata (Lucks, 1912)

T. tetractis (Ehrenberg, 1830) f. similis (Stenroos, 1898)

T. truncata (Whitelegge, 1889)

$\begin{array}{lll}4,5,6 & 4,6,8,9 & \mathrm{Li} \\ 4,5,6 & 8 & \mathrm{Li} \\ 2,3 & 9 & \mathrm{Pe} \\ 2,3,4,5,6 & 4-6,8-10 & \mathrm{Pe}, \mathrm{Li} \\ 2,4,5,6 & 7,8 & \mathrm{Li} \\ 4,5,6 & 6 & \mathrm{Li}\end{array}$

\section{Family Mytilinidae}

Lophocharis naias Wulfert, 1942

L. oxysternon (Gosse, 1851)

Lophocharis sp.

Mytilina mucronata (Müller, 1773)

M. ventralis (Ehrenberg, 1832)

M. ventralis (Ehrenberg, 1832) f. brevispina

(Ehrenberg, 1832)

\section{Family Colurellidae}

Colurella adriatica Ehrenberg, 1831

C. colurus (Ehrenberg, 1830)

C. uncinata (Müller, 1773)

Lepadella acuminata (Ehrenberg, 1834)

L. ovalis (Müller, 1786)

L. patella (Müller, 1773)

L. patella (Müller, 1773) f. biloba Hauer, 1958

L. patella (Müller, 1773) f. oblonga (Ehrenberg, 1834)

$\begin{array}{lll}4,5,6 & 5-7 & \mathrm{Li} \\ 4,5,6 & 8 & \mathrm{Pe}, \mathrm{Li}, \mathrm{Ps} \\ 4,5,6 & 7 & \mathrm{Li} \\ 4,5,6 & 7 & \mathrm{Li} \\ 4,5,6,7 & 4,7,10,12 & \mathrm{Li} \\ 4,5,6 & 6-8 & \mathrm{Li} \\ 4,5,6 & 6 & \mathrm{Li} \\ 4,5,6 & 7 & \mathrm{Li}\end{array}$

\section{Family Euchlanidae}

Dipleuchlanis propatula (Gosse, 1886)

Euchlanis deflexa Gosse, 1851

E. dilatata Ehrenberg, 1832

E. dilatata Ehrenberg, 1832 f. lucksiana Hauer, 1930

E. dilatata Ehrenberg, 1832 f. unisetata Leydig, 1854

E. incisa Carlin, 1939

E. lyra Hudson, 1886

E. lyra myersi Kutikova, 1959

E. oropha Gosse, 1887

E. parva Rousselet, 1892

E. proxima Myers, 1930

E. pyriformis Gosse, 1851

E. triquetra Ehrenberg, 1838

\begin{tabular}{lll}
$4,5,6$ & 4,5 & $\mathrm{Li}$ \\
$2,4,5,6$ & $5,7-9$ & $\mathrm{Li}$ \\
$1,2,4,5,6$ & $6-9$ & $\mathrm{Pe}, \mathrm{Li}$ \\
$2,3,4,5,6$ & $6-10$ & $\mathrm{Pe}, \mathrm{Li}$ \\
$4,5,6$ & $5,6,8,9$ & $\mathrm{Pe}, \mathrm{Li}$ \\
$4,5,6$ & 6 & $\mathrm{Li}$ \\
$4,5,6$ & $4-11$ & $\mathrm{Pe}, \mathrm{Li}$ \\
$4,5,6$ & 7 & $\mathrm{Li}$ \\
$4,5,6,7$ & $2,3,12$ & $\mathrm{Pe}, \mathrm{Li}$ \\
2 & & $\mathrm{Li}$ \\
$2,3,6$ & 5 & $\mathrm{Pe}, \mathrm{Li}$ \\
$4,5,6$ & 7 & $\mathrm{Pe}, \mathrm{Li}$ \\
$2,4,5,6$ & 5,8 & $\mathrm{Li}$ \\
& & \\
$4,5,6$ & $5-9$ & $\mathrm{Pe}, \mathrm{Li}$ \\
$2,3,4,5,6,7$ & $1,3-7,10,12 \mathrm{Pe}, \mathrm{Li}$ \\
6 & $4-6$ & $\mathrm{Pe}$ \\
$4,5,6,7$ & 5,6 & $\mathrm{Li}$ \\
& \multicolumn{3}{c}{ Continued overleaf }
\end{tabular}

Family Brachionidae

Anuraeopsis fissa (Gosse, 1851)

Brachionus angularis Gosse, 1851

B. angularis Gosse, $1851 \mathrm{f}$. bidens Plate, 1886

B. bennini Leissling, 1924

Continued overleaf 
Table 2. Continued

\begin{tabular}{|c|c|c|c|}
\hline Taxon & Source & Month & Habitat \\
\hline B. calyciflorus Pallas, 1766 & $4,5,6,7$ & $1,4-8,10-12$ & $\mathrm{Pe}, \mathrm{Li}$ \\
\hline $\begin{array}{l}\text { B. calyciflorus Pallas, } 1766 \text { f. amphiceros } \\
\text { (Ehrenberg, 1838) }\end{array}$ & $4,5,6$ & $4-6$ & $\mathrm{Pe}, \mathrm{Li}$ \\
\hline B. calyciflorus Pallas, 1766 f. anuraeiformis Brehm, 1909 & $4,5,6,7$ & $4-6,11,12$ & $\mathrm{Pe}, \mathrm{Li}$ \\
\hline B. plicatilis Müller, 1786 f. longicornis Fadeev, 1925 & 6 & 4 & $\mathrm{Pe}$ \\
\hline B. quadridentatus Hermann, 1783 & $1,2,4,5,6$ & $5-8$ & $\mathrm{Pe}, \mathrm{Li}$ \\
\hline $\begin{array}{l}\text { B. quadridentatus Hermann, } 1783 \text { f. brevispinus } \\
\text { Ehrenberg, } 1832\end{array}$ & 6 & 7 & $\mathrm{Pe}$ \\
\hline B. sericus Rousselet, 1907 & $4,5,6$ & 5 & $\mathrm{Pe}, \mathrm{Li}$ \\
\hline B. urceus (Linnaeus, 1758) & $4,5,6$ & $4-6$ & $\mathrm{Pe}, \mathrm{Li}$ \\
\hline Kellicottia longispina (Kellicott, 1879) & $1,2,3,4,5,6,7$ & $1-12$ & $\mathrm{Pe}, \mathrm{Li}$ \\
\hline Keratella cochlearis (Gosse, 1851$)$ & $1,2,3,4,5,6,7$ & $1-12$ & $\mathrm{Pe}, \mathrm{Li}$ \\
\hline $\begin{array}{l}\text { K. cochlearis (Gosse, 1851) f. macracantha } \\
\text { (Lauterborn, 1898) }\end{array}$ & $4,5,6,7$ & $1-6,10$ & $\mathrm{Pe}, \mathrm{Li}$ \\
\hline $\begin{array}{l}\text { K. cochlearis (Gosse, 1851) f. micracantha } \\
\text { (Lauterborn, 1900) }\end{array}$ & 6 & 6,7 & $\mathrm{Pe}$ \\
\hline $\begin{array}{l}\text { K. cochlearis (Gosse, 1851) f. pustulata } \\
\text { (Lauterborn, 1900) }\end{array}$ & 6 & $5-7$ & $\mathrm{Pe}$ \\
\hline K. cochlearis hispida (Lauterborn, 1898) & $1,4,5,6$ & $6-8$ & $\mathrm{Pe}, \mathrm{Li}$ \\
\hline $\begin{array}{l}\text { K. cochlearis hispida (Lauterborn, 1898) f. ecauda } \\
\text { Amman, } 1921\end{array}$ & $4,5,6$ & 7 & $\mathrm{Pe}$ \\
\hline $\begin{array}{l}\text { K. cochlearis hispida (Lauterborn, 1898) f. micracantha } \\
\text { Slonimski, } 1932\end{array}$ & 6 & 6 & $\mathrm{Pe}$ \\
\hline K. cochlearis robusta (Lauterborn, 1900) & 6 & 4,5 & $\mathrm{Pe}$ \\
\hline K. cochlearis tecta (Gosse, 1851) & $1,4,5,6$ & $5-10$ & $\mathrm{Pe}, \mathrm{Li}$ \\
\hline K. hiemalis Carlin, 1943 & $2,3,4,5,6,7$ & $1-6,11,12$ & $\mathrm{Pe}, \mathrm{Li}$ \\
\hline K. irregularis (Lauterborn, 1898) & $4,5,6$ & $5-7$ & $\mathrm{Pe}, \mathrm{Li}$ \\
\hline $\begin{array}{l}\text { K. irregularis (Lauterborn, 1898) f. angulifera } \\
\text { (Lauterborn, 1900) }\end{array}$ & $4,5,6$ & 5,6 & $\mathrm{Pe}$ \\
\hline $\begin{array}{l}\text { K. irregularis (Lauterborn, 1898) f. connectens } \\
\text { (Lauterborn, 1900) }\end{array}$ & 6 & 6,7 & $\mathrm{Pe}$ \\
\hline $\begin{array}{l}\text { K. irregularis (Lauterborn, 1898) f. wartmanni } \\
\text { (Asper et Heuscher, 1889) }\end{array}$ & $4,5,6$ & $6,7,10$ & $\mathrm{Pe}, \mathrm{Li}$ \\
\hline K. quadrata (Müller, 1786) & $1,2,3,6$ & $4-8$ & $\mathrm{Pe}$ \\
\hline K. quadrata (Müller, 1786) f. frenzeli (Eckstein, 1895) & $4,5,6,7$ & $1-12$ & $\mathrm{Pe}, \mathrm{Li}$ \\
\hline K. quadrata (Müller, 1786) f. reticulata Carlin, 1943 & 6,7 & 1,6 & $\mathrm{Pe}$ \\
\hline K. testudo (Ehrenberg, 1832) & $4,5,6$ & 10 & $\mathrm{Pe}$ \\
\hline Notholca acuminata (Ehrenberg, 1832) & $1,2,3,4,5,6$ & $5,6,9,10$ & $\mathrm{Pe}, \mathrm{Li}$ \\
\hline N. caudata Carlin, 1943 & $2,3,6$ & $9-11$ & $\mathrm{Pe}$ \\
\hline N. foliacea (Ehrenberg, 1838) & $2,3,4,5,6$ & 5,6 & $\mathrm{Pe}, \mathrm{Li}$ \\
\hline N. labis Gosse, 1887 & $4,5,6,7$ & $1,5-7,9-11$ & $\mathrm{Pe}, \mathrm{Li}$ \\
\hline N. labis Gosse, 1887 f. limnetica Levander, 1901 & $2,6,7$ & $9-12$ & $\mathrm{Pe}$ \\
\hline N. squamula (Müller, 1786) & $2,3,4,5,6,7$ & $2-5,12$ & $\mathrm{Pe}, \mathrm{Li}$ \\
\hline N. squamula (Müller, 1786) f. evensi Gillard, 1948 & 6 & & $\mathrm{Pe}$ \\
\hline N. squamula (Müller, 1786) f. muelleri Focke, 1961 & 6 & & $\mathrm{Pe}$ \\
\hline N. striata (Müller, 1786) & $1,2,3,6,7$ & $2-5,10-12$ & $\mathrm{Pe}$ \\
\hline Platyias quadricornis (Ehrenberg, 1832) & $2,4,5,6$ & 4,8 & $\mathrm{Li}$ \\
\hline
\end{tabular}


Table 2. Continued

\begin{tabular}{|c|c|c|c|}
\hline Taxon & Source & Month & Habitat \\
\hline \multicolumn{4}{|l|}{ Order Flosculariacea } \\
\hline \multicolumn{4}{|l|}{ Family Flosculariidae } \\
\hline Floscularia sp. & $1,2,6$ & $6,7,10$ & \\
\hline \multicolumn{4}{|l|}{ Family Conochilidae } \\
\hline Conochilus unicornis Rousselet, 1892 & $1,2,3,4,5,6,7$ & $2-9,11$ & $\mathrm{Pe}, \mathrm{Li}$ \\
\hline \multicolumn{4}{|l|}{ Family Testudinellidae } \\
\hline Pompholyx sulcata Hudson, 1885 & $1,2,4,5,6,7$ & 3,6 & $\mathrm{Pe}, \mathrm{Li}$ \\
\hline Testudinella patina (Hermann, 1783) & $1,2,4,5,6$ & $5,7,10$ & $\mathrm{Pe}, \mathrm{Li}$ \\
\hline \multicolumn{4}{|l|}{ Family Trochosphaeridae } \\
\hline Filinia longiseta (Ehrenberg, 1834) & $1,2,3,4,5,6,7$ & $1,3-10,12$ & $\mathrm{Pe}, \mathrm{Li}$ \\
\hline F. limnetica (Zacharias, 1893) & $2,3,6$ & $5-11$ & $\mathrm{Pe}$ \\
\hline F. terminalis (Plate, 1886) & $2,3,4,5,6,7$ & $1-5,7,10,11$ & $\mathrm{Pe}, \mathrm{Li}$ \\
\hline \multicolumn{4}{|l|}{ Order Collothecacea } \\
\hline \multicolumn{4}{|l|}{ Family Collothecidae } \\
\hline Collotheca balatonica Varga, 1936 & $4,5,6$ & $6-11$ & $\mathrm{Pe}, \mathrm{Li}$ \\
\hline C. mutabilis (Hudson, 1885) & $4,5,6$ & $6-11$ & $\mathrm{Pe}, \mathrm{Li}$ \\
\hline C. ornata (Ehrenberg, 1832) & $4,5,6$ & 4 & $\mathrm{Pe}$ \\
\hline Stephanoceros fimbriatus (Goldfuss, 1820) & $4,5,6$ & 8 & $\mathrm{Li}$ \\
\hline
\end{tabular}

The present rotifer community of L. Võrtsjärv is dominated by Anuraeopsis fissa, Keratella cochlearis, K. cochlearis tecta, K. quadrata, Polyarthra dolichoptera, P. luminosa, Synchaeta verrucosa, and Trichocerca rousseleti (Haberman, 1995, 1998; Haberman \& Virro, 2003). The composition of the dominating complex as well as of the whole community is very characteristic of eutrophic lakes in the northern temperate zone (Kutikova, 1970, 1998; Bērziņš, 1978). Typically of the waterbodies of these latitudes, with a quite variable thermal regime, more or less eurythermic taxa constitute the major fraction of the rotifer fauna of the lake. The fully developed winter season (ice cover lasts from November to April) provides a suitable habitat for widely documented (e.g. Carlin, 1943; Berner-Fankhauser, 1983; May, 1983; Bērziņš \& Pejler, 1989b) cold stenotherms such as Filinia terminalis, Keratella hiemalis, Synchaeta lakowitziana, $S$. verrucosa, and thermophobes with somewhat wider thermal tolerance like Notholca squamula and Polyarthra dolichoptera. The winter rotifer assemblage of L. Võrtsjärv is dominated by $P$. dolichoptera, Keratella cochlearis, K. quadrata, and $S$. verrucosa (Virro \& Haberman, submitted). On the other hand, summer water temperature reaching up to $23^{\circ} \mathrm{C}$ as an average (Järvet, 2003), enables besides eurytherms the development of warm stenothermic component including 
Table 3. List of synonyms used in earlier literature for different rotifer taxa encountered in Lake Võrtsjärv

\begin{tabular}{|c|c|}
\hline Synonyms used & Valid name \\
\hline Albertia intrusor Gosse & Albertia naidis Bousfield, 1886 \\
\hline Anuraea aculeata Ehrenberg & Keratella quadrata (Müller, 1786) \\
\hline Anuraea cochlearis Gosse & Keratella cochlearis (Gosse, 1851) \\
\hline $\begin{array}{l}\text { Anuraea cochlearis f. hispida } \\
\quad \text { Lauterborn }\end{array}$ & Keratella cochlearis hispida (Lauterborn, 1898) \\
\hline $\begin{array}{l}\text { Anuraea cochlearis var. tecta } \\
\quad \text { Lauterborn }\end{array}$ & Keratella cochlearis tecta (Gosse, 1851) \\
\hline Argonotholca foliacea (Ehrenberg) & Notholca foliacea (Ehrenberg, 1838) \\
\hline Bipalpus hudsoni (Imhof) & Ploesoma hudsoni (Imhof, 1891) \\
\hline Brachionus bakeri Müller & Brachionus quadridentatus Hermann, 1783 \\
\hline Cathypna luna Ehrenberg & Lecane luna (Müller, 1776) \\
\hline Dinocharis pocillum (Müller) & Trichotria pocillum (Müller, 1776) \\
\hline Dinocharis tetractis Ehrenberg & Trichotria tetractis (Ehrenberg, 1830) \\
\hline Euchlanis lucksiana Hauer & Euchlanis dilatata Ehrenberg, 1832 f. lucksiana Hauer, 1930 \\
\hline Euchlanis myersi Kutikova & Euchlanis lyra myersi Kutikova, 1959 \\
\hline $\begin{array}{l}\text { Filinia longiseta f. limnetica } \\
\quad \text { (Zacharias) }\end{array}$ & Filinia limnetica (Zacharias, 1893) \\
\hline Keratella hispida (Lauterborn) & Keratella cochlearis hispida (Lauterborn, 1898) \\
\hline Keratella hispida f. ecauda Amman & $\begin{array}{l}\text { Keratella cochlearis hispida (Lauterborn, 1898) f. ecauda } \\
\quad \text { Amman, } 1921\end{array}$ \\
\hline $\begin{array}{l}\text { Keratella hispida f. micracantha } \\
\quad \text { Slonimski }\end{array}$ & $\begin{array}{l}\text { Keratella cochlearis hispida (Lauterborn, 1898) f. micracantha } \\
\quad \text { Slonimski, } 1932\end{array}$ \\
\hline Keratella tecta (Gosse) & Keratella cochlearis tecta $($ Gosse, 1851$)$ \\
\hline $\begin{array}{l}\text { Lecane (L.) acronycha Harring et } \\
\text { Myers }\end{array}$ & Lecane curvicornis (Murray, 1913) \\
\hline Lecane (L.) presumpta Ahlstrom & Lecane elsa Hauer, 1931 \\
\hline Lecane luna presumpta Ahlstrom & Lecane elsa Hauer, 1931 \\
\hline Lecane (M.) constricta Murray & Lecane lunaris (Ehrenberg, 1832) \\
\hline Lecane $(M$.$) crenata Harring$ & Lecane lunaris (Ehrenberg, 1832) \\
\hline Lecane (M.) latvica (Bērziņš) & Lecane closterocerca (Schmarda, 1859) \\
\hline Lecane (M.) tethis (Harring et Myers) & Lecane furcata (Murray, 1913) \\
\hline Monostyla cornuta Müller & Lecane cornuta (Müller, 1786) \\
\hline Notholca limnetica (Levander) & Notholca labis Gosse, 1887 f. limnetica Levander, 1901 \\
\hline Notholca longispina Kellicott & Kellicottia longispina (Kellicott, 1879) \\
\hline Polyarthra platyptera Ehrenberg & Polyarthra dolichoptera Idelson, 1925 \\
\hline Polyarthra platyptera Ehrenberg & Polyarthra major Burckhardt, 1900 \\
\hline Polyarthra platyptera Ehrenberg & Polyarthra minor Voigt, 1904 \\
\hline Polyarthra platyptera Ehrenberg & Polyarthra remata Skorikov, 1896 \\
\hline Polyarthra platyptera Ehrenberg & Polyarthra vulgaris Carlin, 1943 \\
\hline $\begin{array}{l}\text { Polyarthra platyptera f. euryptera } \\
\text { Wierzejski }\end{array}$ & Polyarthra euryptera Wierzejski, 1891 \\
\hline Proalides wulferti Sudzuki & $\begin{array}{l}\text { Proalides tentaculatus Beauchamp, } 1907 \text { f. wulferti Sudzuki, } \\
\quad 1959\end{array}$ \\
\hline Pterodina patina Müller & Testudinella patina (Hermann, 1783) \\
\hline $\begin{array}{l}\text { Rattulus capucinus (Wierzejski et } \\
\text { Zacharias) }\end{array}$ & Trichocerca capucina (Wierzejski et Zacharias, 1893) \\
\hline Triarthra longiseta Ehrenberg & Filinia longiseta (Ehrenberg, 1834) \\
\hline Trichotria similis (Stenroos) & Trichotria tetractis (Ehrenberg, 1830) f. similis (Stenroos, 1898) \\
\hline
\end{tabular}


Anuraeopsis fissa, Brachionus quadridentatus, Lecane ludwigii, L. punctata, Polyarthra euryptera, P. longiremis, and Synchaeta stylata (e.g. after May, 1983; Bērziņš \& Pejler, 1989b; De Paggi et al., 2002; Segers, 1995) in the summer assemblage.

The composition of the rotifer community of L. Võrtsjärv reflects expressively its present strongly eutrophic state. Anuraeopsis fissa, Keratella cochlearis tecta, and Trichocerca rousseleti of the dominating complex are generally acknowledged as good indicators of eutrophy (Hakkari, 1972; Pejler, 1983; Bērziņš \& Pejler, 1989a; Ejsmont-Karabin \& Hillbricht-Ilkowska, 1994; Duggan et al., 2001). In the 1960 s, K. cochlearis tecta was occasionally encountered in L. Võrtsjärv as single individuals, whereas A. fissa and T. rousseleti did not become dominant before the 1980s and 1990s (Haberman \& Mäemets, 1973; Haberman, 1998; Haberman \& Virro, 2003). During the last 40 years, the species characteristic of oligo-mesotrophic conditions (Hakkari, 1972; Pejler, 1983; Bērziņš \& Pejler, 1989a; Duggan et al., 2001) have either totally (Asplanchna herrickii) or nearly disappeared (Conochilus unicornis, Kellicottia longispina, Ploesoma hudsoni) following the accelerated eutrophication process in L. Võrtsjärv. The decrease in the abundance of several other species known to prefer lower trophy (Asplanchna girodi, Filinia longiseta, Gastropus stylifer, Keratella hiemalis) has been also observed.

Due to the shallowness of the lake, a strong influence of the wind causing constant resuspension of sediments, and extensive macrophyte zones, the presence of non-planktonic (benthic, littoral, or periphytic) forms (e.g. representatives of the genera Dicranophorus, Cephalodella, Colurella, Lecane, Rotaria, Trichotria) in the pelagial is an expected feature of L. Võrtsjärv.

\section{Comments on selected species}

The rotifer fauna of L. Võrtsjärv includes four rare species:

Cephalodella compacta was originally described from psammon samples from Poland. The subsequent records were from Hungary and North America (Kutikova, 1970; Nogrady et al., 1995). In L. Võrtsjärv, this rare species has been found in densely vegetated psammolittoral in August (Kutikova \& Haberman, 1986).

Eosphora thoides, a benthic or periphytic species, is known from Europe, Australia, and New Zealand (Kutikova, 1970; Nogrady et al., 1995). It has been encountered in the littoral of L. Võrtsjärv in April (Kutikova \& Haberman, 1986).

Monommata grandis is considered a cosmopolitan but rare species with usually single finds in littoral of lentic waters in Europe, Asia, North America, Australia, and Tasmania (Kutikova, 1970; Nogrady et al., 1995). This species is also known as an indicator of oligosaprobic conditions occurring at the $\mathrm{pH}$ range 3.5-9.2 (optimum 5.5) and temperatures $7.3-27.0^{\circ} \mathrm{C}$ (maximum abundance at $17.5^{\circ} \mathrm{C}$ ) (Bērziņš \& Pejler, 1987, 1989b; Nogrady et al., 1995). M. grandis has been reported from the littoral of L. Vorrtsjärv in September (Kutikova \& Haberman, 1986). 
Resticula gelida has been found in the littoral of L. Võrtsjärv in March (Kutikova \& Haberman, 1986). This raptorial benthic rotifer has a cosmopolitan (Europe, Asia, North America, Australia) but rather isolated occurrence in benthos and periphyton of still and slowly flowing waterbodies (Kutikova, 1970; Koste \& Shiel, 1991; Nogrady et al., 1995). R. gelida is usually found in cold water, but is also documented at $17^{\circ} \mathrm{C}$ (Nogrady et al., 1995).

\section{CONCLUDING REMARKS}

In comparison with other waterbodies in Estonia, L. Võrtsjärv is undoubtedly the most thoroughly investigated regarding Rotifera. The remarkably long period of observations, dating back to the $1910 \mathrm{~s}$, with satisfactory monitoring intervals has resulted in a considerable taxonomic list of rotifers. Nevertheless, to date the studies have been mostly restricted to the pelagial region of the lake, which leaves the benthos and littoral zone (incl. periphyton and psammon) poorly investigated. Further studies focussed on these habitats will likely increase the number of taxa. It should be also noted that higher taxonomic resolution is required dealing with illoricate rotifers, especially Bdelloidea.

\section{ACKNOWLEDGEMENTS}

Financial support from the Estonian Ministry of Education and Research (target financed project No. 0362483s03) and Lake Võrtsjärv State Monitoring Programme is gratefully acknowledged. The authors thank two anonymous reviewers for their suggestions and comments on the manuscript.

\section{REFERENCES}

Berner-Fankhauser, H. 1983. Abundance, dynamics and succession of planktonic rotifers in Lake Biel, Switzerland. Hydrobiologia, 104, 349-352.

Bērziņš, B. 1978. Rotatoria. In Limnofauna Europaea (Illies, J., ed.), pp. 54-91. Gustav Fischer Verlag, Stuttgart, New York, Swets \& Zeitlinger B. V., Amsterdam.

Bērziņš, B. \& Pejler, B. 1987. Rotifer occurrence in relation to pH. Hydrobiologia, 147, 107-116.

Bērziņš, B. \& Pejler, B. 1989a. Rotifer occurrence and trophic degree. Hydrobiologia, 182, 171-180.

Bērziņšs, B. \& Pejler, B. 1989b. Rotifer occurrence in relation to temperature. Hydrobiologia, 175, 223-231.

Carlin, B. 1943. Die Planktonrotatorien des Motalaström. Zur Taxonomie und Ökologie der Planktonrotatorien. Medd. Lunds Univ. limnol. Inst., 5, 1-256.

De Paggi, S. J., Radwan, S., Bielanska-Grajner, I., Segers, H., De Smet, W. H., Hollowday, E. D. \& Sanoamuang, L. 2002. Rotifera. Vol. 6: Asplanchnidae, Gastropodidae, Lindiidae, Microcodidae, Synchaetidae, Trochosphaeridae and Filinia. Guides to the Identification of the Microinvertebrates of the Continental Waters of the World 18. Backhuys Publishers, Leiden. 
De Smet, W. H. 1996. Rotifera. Vol. 4: The Proalidae (Monogononta). Guides to the Identification of the Microinvertebrates of the Continental Waters of the World 9. SPB Academic Publishing bv, Amsterdam.

De Smet, W. H. \& Pourriot, R. 1997. Rotifera. Vol. 5: The Dicranophoridae (Monogononta) and the Ituridae (Monogononta). Guides to the Identification of the Microinvertebrates of the Continental Waters of the World 12. SPB Academic Publishing bv, Amsterdam.

Donner, J. 1965. Ordnung Bdelloidea (Rotatoria, Rädertiere). Bestimmungsbücher zur Bodenfauna Europas, 6. Akademie-Verlag, Berlin.

Duggan, I., Green, J. D. \& Shiel, R. J. 2001. Distribution of rotifers in North Island, New Zealand, and their potential use as bioindicators of lake trophic state. Hydrobiologia, 446/447, 155-164.

Ejsmont-Karabin, J. \& Hillbricht-Ilkowska, A. 1994. Illustration of the eutrophication process: comparison of rotifers from Mikołajskie Lake in the years 1989-1990 and 1963-1964. Pol. Arch. Hydrobiol., 41(4), 477-487.

Haberman, J. 1976. An ecological characterization of the rotifers dominating in the pelagic region of lakes Peipsi-Pihkva and Võrtsjärv. In Productivity of Estonian Fresh Waters. Estonian Contributions to the International Biological Programme, $X$ (Haberman, H., Haberman, J. \& Elberg, K., eds.), pp. 35-59. Academy of Sciences of the Estonian SSR, Tartu.

Haberman, J. 1978. Seasonal dynamics of pelagic rotifers of lakes Peipsi-Pihkva and Võrtsjärv. Proc. Acad. Sci. Estonian SSR. Biol., 27, 16-30.

Haberman, J. 1995. Dominant rotifers of Võrtsjärv (Estonia). Hydrobiologia, 313/314, 313-317.

Haberman, J. 1998. Zooplankton of Lake Võrtsjärv. Limnologica, 28(1), 49-65.

Haberman, J. \& Mäemets, A. 1973. Zooplankton. In Võrtsjärv (Timm, T., ed.), pp. 100-113. Valgus, Tallinn.

Haberman, J. \& Põllumäe, A. 1998. Võrtsjärves domineerivad zooplankterid. In Eesti Looduseuurijate Seltsi aastaraamat. 78 (Möls, T., Kalda, A., Kongo, L., Masing, V. \& Milius, A., eds.), pp. 126-154. Estonian Academy Publishers, Tallinn.

Haberman, J. \& Virro, T. 2003. Zooplankton. In Võrtsjärv. Loodus. Aeg. Inimene (Haberman, J., Pihu, E. \& Raukas, A., eds.), pp. 279-299. Eesti Entsüklopeediakirjastus, Tallinn.

Hakkari, L. 1972. Zooplankton species as indicators of environment. Aqua fenn., 1972, 46-54.

Järvet, A. 2003. Võrtsjärve hüdroloogia. In Võrtsjärv. Loodus. Aeg. Inimene (Haberman, J., Pihu, E. \& Raukas, A., eds.), pp. 141-169. Eesti Entsüklopeediakirjastus, Tallinn.

Koste, W. 1978. Rotatoria. Die Rädertiere Mitteleuropas. Überordnung Monogononta. I. Textband, II. Tafelband. Gebrüder Borntraeger, Berlin-Stuttgart.

Koste, W. \& Shiel, R. J. 1987. Rotifera from Australian inland waters. II. Epiphanidae and Brachionidae (Rotifera: Monogononta). Invertebr. Taxon., 7, 949-1021.

Koste, W. \& Shiel, R. J. 1989a. Rotifera from Australian inland waters. III. Euchlanidae, Mytilinidae and Trichotriidae (Rotifera: Monogononta). Trans. r. Soc. S. Aust., 113(1/2), 85-114.

Koste, W. \& Shiel, R. J. 1989b. Rotifera from Australian inland waters. IV. Colurellidae (Rotifera: Monogononta). Trans. r. Soc. S. Aust., 113(3), 119-143.

Koste, W. \& Shiel, R. J. 1991. Rotifera from Australian inland waters. VII. Notommatidae (Rotifera: Monogononta). Trans. r. Soc. S. Aust., 115(3), 111-159.

Kutikova, L. A. 1970. Rotifers (Rotatoria) of the Fauna of the USSR. Eurotatoria (Ploimida, Monimotrochida, Paedotrochida). Nauka, Leningrad (in Russian).

Kutikova, L. A. 1998. Remarks on the rotifer fauna of north and northwestern Russia. Hydrobiologia, 387/388, 79-82.

Kutikova, L. \& Haberman, J. 1986. Rotifers (Rotatoria) of Lake Võrtsjärv. Proc. Acad. Sci. Estonian SSR. Biol., 35, 113-121.

May, L. 1983. Rotifer occurrence in relation to water temperature in Loch Leven, Scotland. Hydrobiologia, 104, 311-315.

Melone, G., Ricci, C., Segers, H. \& Wallace, R. L. 1998. Phylogenetic relationships of phylum Rotifera with emphasis on the families of Bdelloidea. Hydrobiologia, 387/388, 101-107.

Mühlen, M. von zur \& Schneider, G. 1920. Der See Wirzjerw in Livland. Biologie und Fischerei. Archiv für die Naturkunde Liv-, Ehst- und Kurlands II, 14(1), 1-156. 
Nõges, P., Laugaste, R. \& Nõges, T. 2003. Fütoplankton. In Võrtsjärv. Loodus. Aeg. Inimene (Haberman, J., Pihu, E. \& Raukas, A., eds.), pp. 261-277. Eesti Entsüklopeediakirjastus, Tallinn.

Nogrady, T., Pourriot, R. \& Segers, H. 1995. Rotifera. Vol. 3: The Notommatidae and the Scaridiidae. Guides to the Identification of the Microinvertebrates of the Continental Waters of the World 8. SPB Academic Publishing bv, Amsterdam.

Pejler, B. 1983. Zooplanktic indicators of trophy and their food. Hydrobiologia, 101, 111-114.

Schönberg, N. 1958. Angaben über die Futterbasis und Ernährung der planktonfressenden Fische des Sees Wõrtsjärw. In Hydrobiological Researches I (Simm, H., ed.), pp. 191-201. Acad. Sci. Estonian SSR, Tartu (in Russian).

Segers, H. 1995. Rotifera. Vol. 2: The Lecanidae (Monogononta). Guides to the Identification of the Microinvertebrates of the Continental Waters of the World 6. SPB Academic Publishing bv, The Hague.

Shiel, R. J. \& Koste, W. 1992. Rotifera from Australian inland waters VIII. Trichocercidae (Monogononta). Trans. r. Soc. S. Aust., 116(1), 1-27.

Tammert, H. \& Kisand, V. 2003. Bakterplankton. In Võrtsjärv. Loodus. Aeg. Inimene (Haberman, J., Pihu, E. \& Raukas, A., eds.), pp. 249-258. Eesti Entsüklopeediakirjastus, Tallinn.

Virro, T. \& Haberman, J. (Submitted). Winter rotifer assemblages in two large Estonian lakes. Aquat. Ecol.

\section{Võrtsjärve keriloomade annoteeritud nimestik}

\section{Taavi Virro ja Juta Haberman}

Tänapäevasele nomenklatuurile kohandatult on seni avaldatud (alates 1920. aastast) kirjanduse põhjal antud kokkuvõtlik ülevaade Võrtsjärvest leitud keriloomade (hõimkond Rotifera) taksonilisest koosseisust. Lisatud on ka varasemates allikates kasutatud sünonüümide nimekiri. Praeguse seisuga on Võrtsjärvest registreeritud 173 taksonit (138 liiki) keriloomi. Esindatud on 22 sugukonda 46 perekonnaga. Kõige taksonirikkamaks sugukonnaks on Brachionidae 41 taksoniga, järgnevad Synchaetidae 19 ja Trichocercidae 18 taksoniga. Kõige mitmekesisemad perekonnad on Trichocerca ja Lecane: vastavalt 17 ja 14 taksoniga. Enamik (75\%) leitud keriloomadest on kosmopoliitse levikuga või asustavad mitut kontinenti, ligikaudu $11 \%$ taksonitest on levinud Holarktises ja $2 \%$ Palearktises. Viis taksonit (3\%) - Collotheca balatonica, Dicranophorus robustus f. europaeus, Encentrum eurycephalum, Polyarthra dissimulans ja Trichotria pocillum bergiesinevad teadaolevalt ainult Euroopas. Võrtsjärve keriloomade faunasse kuulub ka neli haruldast liiki: Cephalodella compacta, Eosphora thoides, Monommata grandis ja Resticula gelida. Teiste Eesti veekogudega võrreldes on Võrtsjärv keriloomade osas kahtlemata üks põhjalikumalt uuritud veekogusid. Pikka aega (alates 1910. aastatest) - ning selle perioodi kohta rahuldava intervalliga - toimunud vaatluste tulemuseks on küllaltki arvestatav keriloomade nimestik. Sellele vaatamata on suurem osa seniseid uurimusi käsitlenud peaasjalikult pelagiaali, mistõttu bentose ja litoraalivööndi (sh perifüüton ja psammon) keriloomastik on jäänud väheuurituks. Edasiste uuringutega nendest biotoopidest peaks Võrtsjärve keriloomade nimestik tõhusalt täienema. 